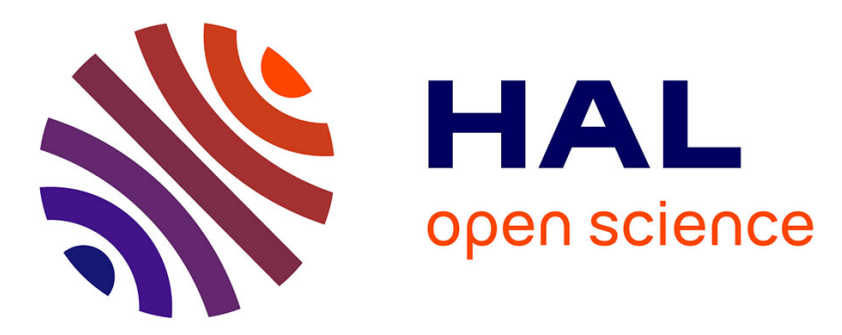

\title{
Les universitaires, combien de divisions ? Lignes de fracture et transformations de la profession académique en France depuis les années 1990
}

\author{
Julien Barrier, Emmanuelle Picard
}

\section{- To cite this version:}

Julien Barrier, Emmanuelle Picard. Les universitaires, combien de divisions ? Lignes de fracture et transformations de la profession académique en France depuis les années 1990. Revue Française de Pédagogie, 2020, 2020/2 (207), pp.19-28. 10.4000/rfp.9146 . halshs-03024766

\section{HAL Id: halshs-03024766 \\ https://shs.hal.science/halshs-03024766}

Submitted on 26 Nov 2020

HAL is a multi-disciplinary open access archive for the deposit and dissemination of scientific research documents, whether they are published or not. The documents may come from teaching and research institutions in France or abroad, or from public or private research centers.
L'archive ouverte pluridisciplinaire HAL, est destinée au dépôt et à la diffusion de documents scientifiques de niveau recherche, publiés ou non, émanant des établissements d'enseignement et de recherche français ou étrangers, des laboratoires publics ou privés. 


\title{
Les universitaires, combien de divisions ? Lignes de fracture et transformations de la profession académique en France depuis les années 1990
}

\author{
Julien Barrier \\ ENS de Lyon, UMR CNRS Triangle, LLE, IFE \\ julien.barrier@ens-lyon.fr
}

\section{Emmanuelle Picard}

ENS de Lyon, UMR CNRS Triangle, LLE, IFE

emmanuelle.picard@ens-lyon.fr

\begin{abstract}
Résumé : Cet article revient sur les transformations de la profession universitaire en France depuis les années 1990, en mettant en évidence une tension structurelle au sein de ce groupe professionnel : alors qu'il s'est historiquement construit autour de l'idéal d'une communauté égalitaire, organisée par des règles uniformes à l'échelle nationale, il a été marqué par une fragmentation croissante au cours des trente dernières années. On a assisté à une recomposition des clivages disciplinaires, une différenciation accrue des conditions d'exercice du métier à l'échelle individuelle et, enfin, une nouvelle stratification des positions de pouvoir dans la profession. En conclusion, les débats engagés en 2019 autour de la Loi de programmation pluriannuelle de la recherche témoignent d'une exacerbation de ces tensions.
\end{abstract}

Mots-clés : France; projet de réforme; profession d'enseignant; enseignement supérieur;

\section{Academics, how many divisions ? The fragmentation of the French academic profession since the 1990s}

\begin{abstract}
This article reviews the transformations of the academic profession in France since the 1990s, highlighting an enduring structural tension : while this group has historically been built around the ideal of an egalitarian community, organized by uniform rules at the national level, it has become increasingly fragmented over the last 30 years. New divisions have developed between and within disciplines, work situations have become increasingly differentiated at the individual level, and a new stratification of power positions has emerged within the profession. In conclusion, the article points out that the debates initiated in 2019 around a proposal to reform the funding and organization of academic research reveal an exacerbation of these tensions.
\end{abstract}

Keywords: France; higher education; reform proposal; teaching profession; 


\section{Introduction}

Novembre 2019. Alors que s'ouvrent les débats sur la future Loi de programmation pluriannuelle pour la recherche (LPPR), le président-directeur général du CNRS Antoine Petit se déclare favorable à une loi « inégalitaire » et «darwinienne », déclenchant aussitôt de vives critiques : chercheurs et enseignants-chercheurs, syndicats, sociétés savantes, collectifs locaux et instances nationales sont alors nombreux à marquer leur opposition à un discours vantant la compétition et la différenciation comme ressorts essentiels de la profession académique. Ces réactions renvoient d'abord aux inquiétudes qui s'expriment depuis une dizaine d'années face à l'intensification de la concurrence et au manque de moyens pour la recherche. Mais elles sont aussi un puissant révélateur de la tension structurelle qui traverse historiquement la profession: bien qu'elle soit depuis longtemps marquée par de multiples clivages, ses membres restent très largement attachés à l'idéal d'une communauté égalitaire, unie autour d'une «République des savants ». De nombreux acteurs redoutent ainsi que les différences de fait ne se transforment en distinctions institutionnalisées, entérinant définitivement l'existence d'un système à plusieurs vitesses ; crainte déjà éprouvée à plusieurs moments de son histoire, mais sans doute aujourd'hui d'une probabilité plus avérée.

Cet article se propose de contribuer aux débats sur la LPPR en revenant sur l'histoire de cette tension entre une différenciation de fait et un idéal d'unité au sein du monde académique. Dans ce cadre, il s'intéresse plus précisément au groupe professionnel que forment les enseignants-chercheurs ${ }^{1}$, en montrant comment cette tension a été exacerbée par la multiplication des clivages internes au sein du groupe depuis les années 1990. Cette situation de tension entre division et unité s'observe dans la plupart des professions (Bucher \& Strass, 1961 ; Champy, 2011) et concerne les enseignants-chercheurs dans de nombreux pays (Clark, 1987). Elle revêt, néanmoins, une intensité probablement sans égal dans le contexte français.

En effet, la profession universitaire s'y est construite depuis deux siècles comme un corps national, partageant le même socle réglementaire et géré par des instances centralisées (Picard, 2012). L'actuel Conseil national des universités (CNU), créé en 1987, est ainsi l'héritier d'une succession de comités aux prérogatives similaires mis en place dès la fin du XIXème siècle. Cette uniformité réglementaire et l'attachement à un principe d'égalité entre agents n'ont jamais empêché le développement de lignes de clivage internes, voire de conflits, entre disciplines, entre universités parisiennes et provinciales, ou entre précaires et titulaires. Pour autant, de la fin du XIXe à la fin du XXe siècle, ces tensions latentes ont généralement été résolues par les possibilités d'ascension individuelle d'une grande partie des membres de la profession, qui pouvaient espérer accéder à de meilleures positions en termes de conditions de travail, de salaire, de pouvoir ou de prestige. Dès lors, l'intensité des tensions a été régulée par l'état du marché du travail académique : faible en période de croissance (fin du XIXe,

\footnotetext{
${ }^{1}$ Nous adoptons ici, par convention, une définition restreinte de la profession universitaire, en n'incluant pas dans nos analyses les chercheurs des organismes tels que le CNRS. Les contraintes de place et de cohérence de cet article ne nous permettront de les mentionner que ponctuellement au fil du texte.
} 
années 1960), forte quand le système se contracte (entre-deux-guerres, années 1950, années 1980).

Or, la croissance du système d'enseignement supérieur au cours de la décennie 1990 marque une rupture dans ce schéma. Avec l'objectif d'amener $80 \%$ d'une classe d'âge au baccalauréat, les réformes scolaires des années 1980 entraînent une augmentation considérable des effectifs étudiants, ouvrant une période faste pour le recrutement d'enseignants-chercheurs, dont le nombre passe d'environ 30000 en 1992 à plus de 50000 en 2002. Si cette massification de l'enseignement supérieur se fait sur un rythme moins rapide que celle des années 1960, elle se traduit par des effets structuraux beaucoup plus importants, qui accentuent les clivages au sein de la profession, avec une différenciation accrue entre établissements, sites géographiques, disciplines, conditions d'exercice du métier ou perspectives de carrière. Depuis le début des années 2000, ce mouvement se trouve amplifié par des politiques nationales qui mettent explicitement l'accent sur les vertus de la différenciation du système, engendrant un décalage croissant entre les représentations historiques de la profession et la réalité de la situation de ses membres. Cette tension ravivée est rendue encore plus aigüe, depuis le début des années 2010, par les contraintes croissantes qui pèsent sur les ressources budgétaires des universités et l'ouverture de postes de titulaires.

En nous appuyant sur des données statistiques et la relecture de nombreux travaux de recherche consacrés à l'enseignement supérieur en France, nous mettons en évidence trois processus qui ont contribué à une fragmentation croissante de la profession universitaire : une recomposition des clivages disciplinaires, une différenciation croissante des conditions d'exercice du métier à l'échelle individuelle et, enfin, un mouvement de stratification hiérarchique au sein de la profession. A la lumière de ces constats, nous reviendrons en conclusion sur les tensions que révèlent les débats sur la future LPPR.

\section{Le poids des disciplines}

Le clivage disciplinaire est le plus évident à documenter. Il apparaît dans la structure même des instances de régulation professionnelle que sont le CNU et le Comité national de la recherche scientifique (CoNRS), tous deux organisés en sections disciplinaires. Tous les travaux sur les enseignants-chercheurs en France soulignent le poids considérable de la variable disciplinaire dans l'explication des différences interindividuelles (Karady, 1983; Bourdieu, 1984 ; Charle, 1994 ; Becquet \& Musselin, 2004 ; Faure \& Soulié, 2005). Il structure historiquement la profession et le système universitaire, comme le montre Kant en 1798 dans son Conflit des facultés, où il oppose les disciplines légitimées par le pouvoir temporel (droit et médecine) à celles fondées sur la seule science (lettres et sciences). Ce principe hiérarchique initial se reconfigure, aux XIXe et XXe siècles, quand émergent et 
s'affirment de nouvelles disciplines au sein des deux entités que constituent les facultés des lettres et les facultés des sciences ${ }^{2}$.

Historiquement, les deux ensembles que constituent les sciences et les lettres - nous dirions aujourd'hui les sciences exactes et expérimentales et les sciences humaines et sociales (SHS) - ont très rapidement après leur création (1808) initié des trajectoires divergentes. D'abord parce que, dès le milieu du XIXe siècle, la recherche s'est imposée comme le critère principal d'évaluation de l'activité professionnelle des membres du premier groupe (Gispert, 1989). Ensuite, parce que les sciences expérimentales ayant accédé au statut d'outil essentiel au développement des sociétés occidentales, leurs représentants ont conquis une position dominante dans les décisions politiques en matière de recherche et d'enseignement supérieur : les physiciens sont ainsi à l'origine de la création du CNRS et jouent un rôle central dans les lobbies en faveur des réformes de la recherche de l'après-guerre (Verschueren, 2017).

La suprématie des sciences se traduit alors concrètement par des investissements plus importants, que ce soit en matière de moyens de recherche ${ }^{3}$ (Picard, 1990) ou d'enseignement (Bettahar \& Choffel-Mailfert, 2014). En cette dernière matière, l'écart se creuse entre lettres et sciences au profit de ces dernières, dès les années 1960. On en retrouve la trace dans le déséquilibre encore sensible aujourd'hui entre les sections «sciences» du CNU qui regroupent encore, en 2018, 24605 enseignants-chercheurs contre 14667 pour les sections « lettres ». Cet écart numérique est d'autant plus sensible qu'il ne reflète en rien des disparités d'effectifs étudiants, voire qu'il leur est symétriquement opposé : dans les années 1960, les planificateurs espéraient une croissance importante du nombre des étudiants en sciences; dans les faits, ce sont les étudiants en lettres et SHS qui se sont multipliés et la tendance ne s'est pas inversée depuis. Il en résulte des taux d'encadrement très différents qui varient en 2000 de 1 enseignant pour 24 étudiants en lettres à un peu plus de 11 étudiants en sciences (Prost \& Cytermann, 2010).

Mais ce principe d'opposition général, s'il reste sensible dans ses grandes lignes, a été bousculé par des recompositions internes et l'émergence de nouvelles disciplines. On voit ainsi se dessiner, depuis les années 1990, une ligne de partage structurée par l'opposition entre disciplines fondamentales et domaines revendiquant une proximité avec le monde socioéconomique au sens large. Ce sont notamment les sciences de l'ingénieur ou les sciences de gestion, mais aussi des disciplines construites autour d'un secteur d'activité professionnel, comme les sciences de l'éducation. D’institutionnalisation plus récente, et longtemps dominées symboliquement, ces disciplines ont bénéficié d'un contexte favorable au développement de formations professionnalisantes, poussées par les pouvoirs publics et plébiscitées par une partie des étudiants (Chambard, 2020 ; Le Cozanet, 2019).

\footnotetext{
${ }^{2}$ Nous nous concentrons ici sur les disciplines héritières des facultés des lettres et des sciences, les problématiques spécifiques à la médecine et au droit rendant la comparaison plus difficile dans le cadre de cet article.

3 On peut donner l'exemple du CNRS, où les SHS ne représentent aujourd'hui que $11 \%$ des effectifs et un institut sur 10 (Bilan social du CNRS, 2016).
} 
Cette redéfinition du poids relatif des disciplines apparaît nettement dans l'évolution des effectifs des sections du CNU entre 1998 et $2018^{4}$. En effet, les plus fortes croissances s'observent notamment en sciences de l'éducation (+ $51 \%)$, sciences de l'information et de la communication $(+76 \%)$, sciences et techniques des activités physiques et sportives (+193\%), informatique (+53\%), génie mécanique et génie informatique $(+42 \%$ et $+43 \%)$. A l'inverse, les disciplines canoniques, fragilisées, voient leurs effectifs stagner, voire diminuer : langues anciennes (-16\%), allemand (-33\%), mathématiques et les trois sections de physique (-15\% en moyenne). L'ouverture ou le redéploiement de postes vers les nouvelles disciplines ont été motivés par l'afflux d'étudiants mais aussi, dans certains cas, par des politiques favorisant l'essor de recherches orientées vers l'innovation et le développement technologique (Barrier, 2014). Cela est visible au CNRS où, entre 2000 et 2011, on constate une baisse de $7 \%$ des effectifs de chercheurs en physique fondamentale (sections 2 à 6), contre une hausse de $28 \%$ de ceux en sciences de l'ingénieur (section 7 à 10) ${ }^{5}$.

Mais les différences entre les disciplines ne se réduisent pas à une simple divergence en termes de dynamique démographique. On observe, en parallèle et sur la longue durée, l'affirmation d'ethos disciplinaires spécifiques, caractérisés par des pratiques distinctes et la délimitation de territoires juridictionnels, selon un processus d'institutionnalisation des disciplines académiques qui s'observe dans tous les pays occidentaux depuis la fin du XIXe siècle. Dans le contexte français, cette différenciation s'est adossée à la mise en place d'instances nationales de régulation (CNU ou CoNRS) dont l'organisation en sections disciplinaires a suivi un découpage de plus en plus fin, au fur et à mesure de la reconnaissance de nouveaux domaines. (Picard, 2020). Chaque discipline fonctionne ainsi, au niveau national, comme un collectif inscrit dans un cadre règlementaire unifié, mais jouissant d'une grande autonomie dans ses pratiques de gestion. On peut en donner pour exemple les taux de qualification aux fonctions de maîtres de conférences selon les sections du CNU, qui varient de $20 \%$ à plus de $85 \%$, indépendamment du nombre des candidats ${ }^{6}$. Les différences s'observent aussi dans les pratiques, qu'elles soient d'enseignement (disciplines appliquées enseignées dans une perspective professionnalisante versus formations fondamentales), avec les différences de publics étudiants qu'on y observe (Renisio, 2015), ou de recherche (sciences expérimentales ou de terrain, plus collectives et consommatrices de moyens financiers versus disciplines théoriques). Différentes études montrent systématiquement la place que tient la discipline de l'enseignant-chercheur dans les caractéristiques de son expérience professionnelle (Becquet \& Musselin, 2004 ; Faure \& Soulié, 2005 ; David, 2015 ; Bodin et al., 2018).

L'affirmation de la division de la profession en collectifs disciplinaires se produit conjointement avec une remise en cause de la hiérarchie canonique des disciplines, telle

\footnotetext{
4 Ces données sont tirées des données publiées par le Ministère de l'enseignement supérieur (https://www.enseignementsup-recherche.gouv.fr/cid85019/fiches-demographiques-dessections-du-cnu.html ).Un tableau complet est disponible dans les annexes électroniques de cet article. 5 Bilan social du CNRS, 2012- données accessibles à http://bilansocial.dsi.cnrs.fr/2012/public/page/permanent

${ }^{6}$ Le ministère publie chaque année un bilan de la campagne de qualification qui fait apparaître les taux par section.
} 
qu'elle a pu être théorisée par Bourdieu par exemple (1984). Paradoxalement, l'autonomie de gestion professionnelle telle qu'elle s'observe au CNU (et donc la possibilité de légitimer des pratiques spécifiques) a permis la remise en cause de la suprématie du pôle fondamental, qui avait imposé ses normes durant tout le XXe siècle, s'appuyant sur le dynamisme de nouvelles disciplines plus appliquées. Dans ce contexte, les fusions d'universités, en regroupant dans un même établissement des groupes de disciplines longtemps séparés, contribuent aussi à les placer dans une concurrence frontale dans une période de réduction des moyens (Charle, 2020).

\section{Une différenciation des conditions d'exercice du métier d'universitaire}

De fait, la discipline n'est plus la variable unique, voire déterminante, des variations au sein de la profession académique. Depuis la fin des années 1980, les conditions d'exercice du métier d'universitaire ont connu une différenciation croissante, non seulement entre établissements, mais aussi entre individus appartenant au même établissement, produite par un contexte en rapide transformation.

Cette évolution résulte, en premier lieu, de la massification de l'enseignement supérieur dans les années 1990, caractérisée par une hausse des effectifs étudiants et une diversification inédite de leur profil social et scolaire, avec une plus grande proportion de jeunes d'origine populaire et de bacheliers des filières technologiques ou professionnelles (Aschieri, 2013). Cette croissance se traduit par la multiplication des missions confiées aux universitaires et l'augmentation de nombreuses tâches qui représentent un «travail invisible » (Losego, 2004), comme la coordination des équipes enseignantes, le développement de formations professionnalisantes ou l'accompagnement d'étudiants aux profils disparates, très inégalement préparés à réussir dans l'enseignement supérieur.

Alors que ces activités sont indispensables au bon fonctionnement des formations, elles sont dévalorisées au regard de l'investissement dans la recherche qui représente depuis longtemps le principal facteur de reconnaissance et de progression dans la carrière (Faure et al., 2008). Or, le poids de ces tâches est très variable en fonction des filières et des établissements, des effectifs enseignants et du profil social et scolaire de leurs étudiants, venant complexifier les facteurs de différenciation déjà évoqués pour les disciplines. En tendance, là où certaines grandes écoles et universités les plus prestigieuses sont restées des lieux privilégiés pour la recherche, les établissements plus récents ont été absorbés par leurs missions d'enseignement. Dans ce contexte, la création de nouvelles universités, comme celle de Marne-la-Vallée en 1991, l'ouverture de nombreux IUT et la multiplication des implantations universitaires délocalisées (les «antennes ») ont amplifié et institutionnalisé une différenciation des situations individuelles (Felouzis, 2001). Une forme de spécialisation régionale semble ainsi se dessiner : les établissements dont l'activité principale se situe dans les premiers cycles et 
les filières professionnalisantes se concentrant dans le quart nord-est de la France quand ceux qui accueillent les $3^{\mathrm{e}}$ cycles sont majoritaires en région parisienne (Baron, 2010) ${ }^{7}$.

Certes, l'existence de tensions entre différentes facettes du métier, tout particulièrement entre recherche et enseignement, n'est pas une nouveauté (Endrizzi, 2017). Pour autant, de par l'ampleur des différences qui se mettent en place, les années 1990 marquent sans doute une fragmentation inédite des conditions de travail des universitaires. Difficile à mesurer, cette évolution transparaît dans la multiplication de rapports consacrés au métier d'enseignantchercheur, alors que cette question avait rarement fait l'objet de débats jusqu'à cette période. Signe de la montée de ces préoccupations, un rapport parlementaire n'hésite pas à affirmer : « Les universitaires forment une communauté scientifique extrêmement peu homogène [...] Plus que de communauté, il faudrait parler d'une juxtaposition de corps distincts aux préoccupations différentes » (Fréville, 2001, p. 14).

A partir des années 2000, ces différenciations de fait se sont doublées d'une hiérarchisation de plus en plus explicite du système, principalement articulée autour de la recherche. Ce mouvement repose d'abord sur l'autonomie croissante des établissements vis-à-vis de l'Etat, consacrée par la loi Libertés et responsabilités des universités de 2007, qui les a de plus en plus poussés à développer leurs propres stratégies et axes de spécialisation. Mais il prend toute son ampleur à partir du Plan Campus et, surtout, des « politiques d'excellence » lancées à la fin des années 2010 (Musselin, 2017). Alors que les politiques nationales avaient longtemps mis l'accent sur les équilibres régionaux, ces dispositifs fondés sur des appels d'offres sélectifs visent à faire émerger un nombre restreint de pôles de recherche structurés autour «d'universités de classe mondiale » (Aust et al., 2018). Longtemps implicite, la différenciation des établissements par la recherche tend ainsi à s'institutionnaliser, comme en témoigne la diffusion de la notion «d'université de recherche intensive », réservée à une poignée d'institutions.

Ce mouvement de concentration des ressources sur certains établissements, et sur certains laboratoires en leur sein, amplifie encore les inégalités entre enseignants-chercheurs. Ses effets sont d'autant plus importants que les modes de financement et d'évaluation de la recherche ont aussi connu de profondes évolutions : ils ont ainsi contribué à instaurer des différences plus marquées entre individus et à les classer en fonction de leurs «performances scientifiques ».

La diffusion rapide à la fin des années 2000 d'indicateurs de publication, de mesure des citations et de classement de revues en est l'un des traits les plus visibles. Certes, ces indicateurs ont souvent été critiqués pour leur caractère réducteur (Gozlan, 2015) et diversement utilisés en fonction des disciplines (Pontille \& Torny, 2012). Néanmoins, ils ont participé à faire exister publiquement une hiérarchisation autrefois implicite des chercheurs et des laboratoires et, surtout, à en banaliser le principe. Pendant la même période, le recours à

\footnotetext{
7 De même, au sein d'une même région, les flux étudiants font apparaître une polarisation des établissements en fonction de leur attractivité et des profils sociaux de leurs publics (Frouillou et Moulin, 2019).
} 
des appels à projets pour financer les activités de recherche des universitaires, dont le principe remonte aux années 1960 (Aust \& Picard, 2014), est devenu beaucoup plus massif et systématique (Barrier, 2011). Or, même si certains laboratoires s'efforcent de mutualiser une partie de leurs moyens (Louvel, 2011), ce mode de financement tend à créer des disparités de ressources entre chercheurs. Il concourt également à établir une nouvelle forme de reconnaissance symbolique au sein de la profession, fondée sur la capacité des individus à obtenir des fonds (Jouvenet, 2011). Ainsi, plus un financement est jugé sélectif, plus la reconnaissance est importante, comme le montre la publicité considérable donnée aux chercheurs lauréats de bourses de l'European research council, qui peuvent aussi bénéficier de primes financières individuelles.

Ces multiples formes de différenciation entre individus tendent à se cumuler et à engendrer des déséquilibres durables au fil de leur carrière (Musselin, 2015). La place des femmes au sein de la profession académique en est emblématique. Elles souffrent non seulement d'un accès plus faible à la profession, en particulier en sciences, mais ensuite tout au long de la carrière, avec des promotions plus lentes et des investissements collectifs moins bien rétribués (Rogers \& Molinier, 2016).

Si les disciplines restent un élément clivant essentiel, il n'en reste pas moins que les transformations d'ensemble du système, croissance et différenciation, ont contribué à rebattre les cartes et à produire une individualisation croissante des expériences professionnelles des enseignants-chercheurs. La taille de l'établissement, son ancienneté, la proximité ou l'éloignement des équipes ou des équipements, le poids des responsabilités administratives, ou encore les stratégies de carrière individuelles qui tiennent compte de tous ces éléments (Louvel \& Valette, 2014), en constituent des facteurs importants. Ils sont la source d'inégalités parfois très fortes entre des pairs, d'autant plus difficiles à vivre que ceux-ci sont par ailleurs régis par des dispositifs réglementaires identiques, en matière de recrutement, de promotion, de salaire et d'obligations statutaires. De plus, à cette atomisation croissante de la profession s'ajoute une nouvelle forme de stratification hiérarchique entre ses membres.

\section{Une nouvelle stratification de la profession}

Historiquement, la profession universitaire s'est construite autour d'un idéal de collégialité et d'égalité entre pairs qui, en principe, s'oppose à l'exercice d'une autorité strictement hiérarchique. Bien sûr, cela n'a jamais empêché l'existence d'une stratification interne au monde universitaire, liée à des différences de statut administratif et à l'occupation de positions de pouvoir, dont la critique était déjà au cœur des mouvements de Mai 1968 (Gaubert et Pouly, 2012). Cependant, on a assisté à une profonde recomposition des formes de cette stratification depuis les années 1990.

On observe, en premier lieu, une redéfinition de la division du travail entre différentes catégories de personnels universitaires, dont les relations avaient connu de fortes tensions dans les années 1970. Dans le sillage de la loi Savary de 1984 sur le statut des enseignants- 
chercheurs, le fonctionnement des universités a vu s'atténuer les tensions entre les personnels dits de rang $\mathrm{A}$, les professeurs, et ceux de rang $\mathrm{B}$, les maîtres de conférences (antérieurement maîtres assistants). Certes, les premiers disposent toujours de prérogatives spécifiques et d'une place généralement plus enviable dans la division du travail: en tendance, ils enseignent moins en premier cycle que les maîtres de conférences et prennent en charge des responsabilités administratives plus valorisées (Becquet \& Musselin, 2004). Néanmoins, le clivage est beaucoup moins marqué que par le passé, les rang B étant davantage impliqués dans les processus de décision et les responsabilités dont ils étaient auparavant largement exclus. Amorcé dans les années 1980, ce mouvement s'est renforcé dans le domaine de la recherche au cours des années 2000, quand les maîtres de conférences ont été de plus en plus incités à s'investir dans la recherche de financements pour faire face à une pression accrue sur le budget des laboratoires.

En revanche, la ligne de clivage entre personnels titulaires et non titulaires, notamment les doctorants et post-doctorants aspirant à une carrière universitaire, est repassée au premier plan après une éclipse durable. Cet enjeu avait été au cœur des débats des années 1970, lorsque de nombreux contractuels protestaient contre le déclin des perspectives de recrutement et de progression de carrière. La vague de titularisation des chercheurs contractuels (les « hors statut ») du début de la décennie 1980, l'intégration des assistants dans le nouveau corps des maîtres de conférences à partir de 1984, puis la croissance des effectifs d'enseignantschercheurs des années 1990, avaient rendu ce clivage moins prégnant. Il est revenu au centre de l'actualité depuis la fin des années 2000.

En accédant à l'autonomie et en devenant responsables de la gestion de leur masse salariale, les universités ont été contraintes de réaliser des économies drastiques sur le recrutement de titulaires : le nombre de postes de maîtres de conférences et de professeurs ouverts chaque année a diminué de $40 \%$ en 10 ans (3 270 en 2008 pour 1986 en 2018) ${ }^{8}$. Dans un contexte où les effectifs étudiants n'ont cessé de croître, cette évolution n'a pu être compensée que par le recours croissant à des enseignants non-titulaires : entre 1999 et 2007, ils représentaient $20 \%$ du corps enseignant (Schwartz, 2008); en 2017, cette proportion atteint $25 \%$ (MESRI, 2019). Une part croissante des enseignements est donc assurée par des personnels précaires, catégorie qui regroupe cependant des situations très variées, aussi bien en termes de statut (doctorants contractuels, professionnels extérieurs, vacataires chargés de cours) que de conditions de travail, de délais de versement des salaires, de types d'enseignement à prendre en charge ou d'insertion dans les équipes pédagogiques.

En matière de recherche, on constate également un recours croissant au personnel précaire dans le cadre du développement des recherches sur projet, à la fois en tant que chercheurs en post-doctorat ou que gestionnaires de projets (Harroche, 2019). En même temps qu'elle accroit le recours à des non-titulaires, la réduction du nombre de postes ouverts au concours rend plus incertain leur accès à un emploi stable. La période de précarité, traditionnellement

\footnotetext{
8 Toutes ces données sont accessibles en ligne sur le site du MESRI, dans ses publications sur les bilans annuels des campagnes de recrutement des enseignants-chercheurs. Il s'agit des postes sur emplois d'Etat.
} 
acceptée malgré ses difficultés au motif qu'elle constituait une étape préalable à la poursuite d'une carrière académique, tend à devenir une épreuve de longue durée et pleine d'incertitudes (Calmand et al. 2017). Dans ces conditions, les tensions se sont intensifiées entre titulaires et précaires.

A l'opposé du spectre professionnel, une nouvelle ligne de clivage s'est dessinée, avec l'émergence progressive d'une strate d'universitaires spécialisés dans des fonctions managériales, notamment dans la direction des établissements. Si leur influence personnelle est sans doute moins étendue que celle des mandarins des années 1960, ces acteurs se caractérisent par de nouvelles manières d'exercer un pouvoir sur leurs pairs, qui prennent un tour plus managérial : il ne s'agit plus simplement d'allouer des ressources dans le secret d'un comité, mais de définir des normes d'évaluation, des règles de gestion ou des schémas organisationnels qui viendront encadrer le travail des autres universitaires, en s'appuyant de plus en plus sur des experts de la gestion (Barrier \& Musselin, 2015). Ces changements de pratiques s'accompagnent d'une polarisation des valeurs au sein de la profession: les universitaires occupant des responsabilités administratives élevées adhèrent davantage que leurs collègues à des principes de mise en concurrence et de mesure de la performance (Chatelain-Ponroy et al., 2018).

Cette différenciation est plus ou moins marquée suivant le niveau de responsabilité et les fonctions exercées. Le fonctionnement des agences de financement et d'évaluation s'est aligné sur des normes plus managériales, mais les universitaires qui y travaillent restent généralement proches des préoccupations de leurs collègues et ne font pas nécessairement carrière dans ces fonctions. De fait, une partie des universitaires qui se sont engagés dans la refonte des structures d'évaluation de la recherche, à la fin des années 2000, l'ont justement fait pour défendre l'autonomie de la profession face à de possibles dérives managériales (Gozlan, à paraître).

Le décalage s'est, par contre, nettement accentué entre les universitaires dirigeant un établissement et leurs collègues. Longtemps conçu comme une activité exercée à temps partiel, parfois purement honorifique, le rôle de président d'université s'est de plus en plus inscrit dans une trajectoire de spécialisation vers des fonctions administratives. Tandis que l'accès à la fonction a nécessité l'acquisition de compétences gestionnaires et politiques spécifiques, les présidents se sont orientés plus fréquemment vers d'autres postes administratifs à l'issue de leur mandat (Bordes, thèse en cours). Outre les postes de recteurs et de conseillers ministériels, la multiplication de nouvelles structures, locales ou nationales, crée un appel d'air pour des universitaires aguerris, susceptibles d'y exercer des fonctions de pilotage. Proches de la haute fonction publique, certains anciens présidents ont acquis une position centrale dans la définition des politiques nationales de l'enseignement supérieur et de la recherche, où ils promeuvent des choix favorables à un renforcement de la stratification de la profession (Gally, 2018).

Ces tendances dessinent donc le portrait d'un groupe stratifié par une hiérarchie de statuts et de fonctions administratives, marqué à ses extrêmes d'une part par l'essor d'une élite managériale issue des rangs de la profession et, d'autre part, par un groupe de travailleurs plus 
ou moins précaires, mais indispensables pour assurer les missions de base des universités. Ce constat fait écho à ce qu'on observe depuis longtemps dans d'autres groupes professionnels, comme les médecins (Freidson, 1984). Néanmoins, la situation reste encore sensiblement différente de celle d'autres pays occidentaux, comme le Royaume-Uni, où s'exerce une division du travail beaucoup plus poussée au sein de la profession académique, entérinée par des statuts et des dispositifs de gestion des carrières (Paye, 2015). On touche là à l'une des particularités du monde universitaire français, historiquement dominé par une vision de l'enseignement supérieur comme un service public national, égalitaire et unifié, qui entre en tension avec la différenciation croissante des situations de travail.

\section{Au-delà des clivages, une réaffirmation de l'unité de la profession ?}

Les discussions autour du futur projet de LPPR durant l'hiver 2019-2020 ont été l'occasion de voir rejouer l'ensemble de ces clivages, avec des réactions plus ou moins critiques en fonction des disciplines, des lieux d'exercice et des positions professionnelles occupées.

A cette occasion, on a vu notamment ressurgir la ligne de fracture qui sépare les SHS des sciences expérimentales, et que l'on a pu observer à différentes reprises ces vingt dernières années. En 2009, la dénonciation des effets pervers de la modulation des services d'enseignement, prévue dans le décret de réforme du statut des enseignants-chercheurs, avait émané des juristes et des SHS, bien plus que des sciences expérimentales (Paradeise, 2011). De même, les universitaires en SHS se montrent plus critiques face aux transformations de la recherche, en particulier au sujet des financements sur projet: ils en condamnent fréquemment le principe lui-même, là où leurs collègues de sciences expérimentales pointent plutôt les limites du dispositif (Giry \& Schultz, 2017). La position moins critique, en tendance, des sciences expérimentales s'explique aisément par les éléments historiques déjà évoqués: l'importance précoce des financements sur projet, les taux d'encadrement des étudiants plus favorables, ainsi que la plus grande implication de leurs représentants dans la construction des politiques de la recherche. A l'inverse, les juristes et les SHS ont plus à perdre, au sens d'une remise en cause de leurs pratiques et d'une fragilisation supplémentaire de leurs conditions d'exercice du métier.

Cependant, au-delà de la variété des réactions aux différentes modalités de transformation possibles du système, il est frappant de constater l'existence d'un mouvement convergent de contestation. Transcendant les oppositions disciplinaires, les prises de position collectives de l'ensemble des sections du CoNRS et du CNU visent à dénoncer les restrictions budgétaires et des contraintes accrues. En matière d'enseignement, la conjonction problématique de la baisse régulière du nombre de postes au recrutement et de la croissance tout aussi régulière des effectifs étudiants s'est vue alourdie par le redéploiement des personnels administratifs de soutien vers les services centraux des établissements, pour faire face à leurs nouvelles prérogatives de gestion budgétaire et de pilotage. La pression est également forte sur la recherche, comme l'atteste la baisse du taux de succès aux demandes de financement de projets adressées à l'Agence nationale de la recherche, passé de $25 \%$ à $10 \%$ entre 2007 et 
2014, avant de remonter à $16 \%$ en 2018. La situation s'est tendue jusque dans les disciplines a priori les moins défavorisées, comme le souligne une enquête quantitative sur les sciences de l'ingénieur, pourtant au cœur des priorités gouvernementales : une large part des titulaires recrutés depuis la fin des années 2000 y éprouve un réel malaise professionnel, alors même qu'ils sont encore en début de carrière et bénéficient d'un emploi stable (Conseil scientifique de l'INSIS, 2018).

Ce contexte a un effet paradoxal : il tend simultanément à raviver les clivages internes à la profession et à favoriser un sentiment d'unité autour de la dénonciation d'une dégradation généralisée du métier. Fragilisée, la profession fait corps pour revendiquer une augmentation des moyens consacrés à ses missions, mais aussi pour défendre les dispositifs qui garantissent a minima une égalité formelle dans les statuts et une uniformité des modes de régulation à l'échelle nationale. De fait, l'hypothèse d'une suppression du CNU, un temps évoquée, est l'un des éléments qui a suscité les réactions les plus vives. L'attachement à une gestion et une régulation professionnelles centralisées, construit dans la longue durée, constitue une valeur fondamentale largement partagée, par-delà les clivages, par une partie significative de la profession.

De ce point de vue, si les universitaires sont confrontés dans de nombreux pays occidentaux à des contraintes comparables, avec une multiplication des fractures au sein de la profession (voir par exemple Finkelstein et al., 2016), la tension entre unité et division du groupe revêt une forme particulière dans le cas français. A l'heure actuelle, celui-ci se caractérise par la coexistence, d'un côté, de facteurs qui intensifient la concurrence et individualisent les conditions d'exercice du métier et, d'un autre côté, d'un ensemble de dispositifs qui maintiennent, bon an mal an, une certaine égalité de traitement et de statut à l'échelle nationale. Renvoyant à des pratiques et des normes profondément institutionnalisées, la défense de ces dispositifs revêt une dimension à la fois matérielle et symbolique. En effet, audelà des garanties règlementaires qu'ils apportent, ils incarnent une représentation égalitaire et unifiée du métier. Celle-ci constitue un facteur d'identification et de cohésion d'autant plus puissant pour les universitaires français que les conditions concrètes d'exercice de leur travail s'éloignent d'une vision idéalisée du métier et poussent à une fragmentation toujours plus grande de leur profession.

\section{$\underline{\text { Bibliographie }}$}

Aschieri Gérard (2013). «Les inégalités dans l'enseignement supérieur ». Le français aujourd'hui, $\mathrm{n}^{\circ} 183$, p. 51-59.

Aust Jérôme \& Emmanuelle Picard (2014). « Gouverner par la proximité. Allouer des fonds à des projets de recherche dans les années 1960 ». Genèses, n 1, 2014, p. 7-31

Aust Jérôme, Mazoyer Harold \& Musselin Christine (2018). « Se mettre à l'IDEX ou être mis à l'index. Conformations, appropriations et résistances aux instruments d'action publique dans trois sites d'enseignement supérieur ». Gouvernement et action publique, n 7(4), p. 9-37. 
Baron Myriam (2010). «Les transformations de la carte universitaire depuis les années 1960 : constats et enjeux ». Le Mouvement Social, $\mathrm{n}^{\circ}$ 233, p. 93-105.

Barrier Julien \& Musselin Christine (2015). « La réforme comme opportunité professionnelle : autonomie des établissements et montée en puissance des cadres administratifs des universités ». Gouvernement et action publique, ${ }^{\circ}$ 4(4), p. 127-151.

Barrier Julien (2011). «La science en projets : financements sur projet, autonomie professionnelle et transformations du travail des chercheurs académiques », Sociologie du Travail, Elsevier Masson, $\mathrm{n}^{\circ} 53(4), \mathrm{p} .515-536$.

Barrier Julien (2014). « Partenaires particuliers : financements sur projet et travail relationnel dans les réseaux de collaboration science-industrie ». Genèses, n ${ }^{\circ}$ 94, p. 55-80.

Becquet Valérie \& Musselin Christine (2004). Variations autour du travail des universitaires. Rapport produit dans le cadre d'une convention MENRT 2002-2007 sur le travail des universitaires.

Bettahar Yamina \& Choffel-Mailfert Marie-Jeanne (dir.) (2014). Les universités au risque de l'histoire. Principes, configurations, modèles. Nancy : Presses universitaires de Nancy/Éditions universitaires de Lorraine.

Bodin Romuald, Millet Mathias \& Saunier Émilie (2018). « Entre triple contrainte et ancrage disciplinaire. Pratiques et conditions d'enseignement à l'Université ». Cahiers de la recherche sur l'éducation et les savoirs, ${ }^{\circ} 17$, p. 143-167.

Bordes Etienne (en cours). La Conférence des présidents d'université (1971-2007). Une socio-histoire du gouvernement de l'université en France. Thèse en cours, université Toulouse 2 Le Mirail.

Bourdieu Pierre (1984). Homo Academicus. Paris : Minuit.

Bucher Rue \& Strauss Anselm (1961). «Professions in process ». American journal of sociology, vol. 66(4), p. 325-334.

Calmand Julien, Prieur Marie-Hélène \& Wolber Odile (2017). Les débuts de carrière des docteurs : une forte différenciation des trajectoires professionnelles. Céreq Bref, $\mathrm{n}^{\circ} 354$.

Chambard Olivia (2020). Business model. L'université nouveau laboratoire de l'idéologie entrepreneuriale. Paris : La Découverte.

Champy Florent (2011). Nouvelle théorie sociologique des professions. Paris : Presses Universitaires de France.

Charle Christophe (1994). La République des universitaires, Paris : Seuil.

Charle Christophe (2020). «À la conquête du «meilleur des mondes »- à propos de la Loi de programmation pluriannuelle sur la recherche (LPPR) », AOC : Analyse, Opinion, Critique, février.

Chatelain-Ponroy Stéphanie, Mignot-Gérard Stéphanie, Musselin Christine \& Sponem Samuel (2018), «Is Commitment to Performance-based Management Compatible with Commitment to University "Publicness"? Academics' Values in French Universities ». Organization Studies, n 39(10), p. 13771401.

Clark Burton (1987). The Academic Life: Small Worlds, Different Worlds. Princeton : Princeton University Press.

Conseil scientifique de l'INSIS (2018), Contribution à l'analyse des conditions d'intégration des jeunes chercheurs dans les laboratoires rattachés à l'INSIS, CNRS et Comité national de la recherche scientifique. 
David Marie (2015). «Les savoirs des formes de scolarisation. Comparaison entre le lycée et la première année de licence ». Revue française de pédagogie, n 193, p. 25-40.

Endrizzi Laure (2017), « Recherche ou enseignement : faut-il choisir ? », Dossier de veille de l'IFÉ, $\mathrm{n}^{\circ} 116$.

Faure Sylvia \& Soulié Charles (2005). Enquête exploratoire sur le travail des enseignantschercheurs : vers un bouleversement de la table des «valeurs académiques »?

Faure Sylvia, Soulié Charles \& Millet Mathias (2008). «Visions et divisions à l'université ». Recherche et formation, $\mathrm{n}^{\circ}$ 57, p. 79-87.

Felouzis Georges (2001). «Les délocalisations universitaires et la démocratisation de l'enseignement supérieur ». Revue française de pédagogie, vol. 136, p. 53-63.

Finkelstein Martin, Martin Conley Valerie \& Schuster Jack H. (2016). The faculty factor: Reassessing the American academy in a turbulent era. Johns Hopkins University Press.

Freidson Eliott (1984). «The Changing Nature of Professional Control ». Annual Review of Sociology, vol. 10, p. 1-20.

Fréville Yves (2001), Rapport d'information sur la politique de recrutement et la gestion des universitaires et des chercheurs, ${ }^{\circ} 54$, Sénat.

Frouillou Leïla \& Moulin Léonard (2019). « Les trajectoires socialement et spatialement différenciées des étudiants franciliens », Formation emploi, n 145 , p. 7-28..

Gally Natacha (2018), «Entre executive shift et gouvernement à distance. La genèse des politiques « pour l'excellence » dans le secteur de l'enseignement supérieur et de la recherche (2009-2012) », Revue française de science politique, Vol. 68, $\mathrm{n}^{\circ} 4$, p. 691-715

Gaubert Christophe \& Pouly Marie-Pierre (2012). «Transformations morphologiques et mobilisations disciplinaires : Les enseignants et étudiants de l'Institut d'anglais de la Sorbonne en $1968 »$. Actes de la recherche en sciences sociales, $\mathrm{n}^{\circ} 194, \mathrm{p} .78-97$.

Giry Johann \& Schultz Emilien (2017). «L'ANR en ph (r) ase critique. Figures et déterminants de la critique d'un dispositif de financement », Zilsel, n², p. 63-96.

Gispert Hélène (1989), «L'enseignement scientifique supérieur et ses enseignants, 1860-1900 : les mathématiques », Histoire de l'éducation, ${ }^{\circ}$ 41, p. 47-78.

Gozlan Clémentine (2015), «L'autonomie de la recherche scientifique en débats : évaluer l'"impact" social de la science ?", Sociologie du travail, n 2 , p.151-74.

Gozlan Clémentine (à paraître), Les valeurs de la science. Enquête sur l'évaluation de la recherche en France, Lyon, ENS Editions.

Harroche Audrey (2019). «Les petites mains de l'excellence. Place et rôle des chargées de projet dans la mise en œuvre d'une Initiative d'excellence », Revue française d'administration publique, vol. 169, no. 1, p. 151-167.

Jouvenet Morgan (2011), «Profession scientifique et instruments politiques : l'impact du financement « sur projet » dans des laboratoires de nanosciences », Sociologie du travail, n 2, p. 234-252.

Karady Victor (1983), «Les professeurs de la République : Le marché scolaire, les réformes universitaires et les transformations de la fonction professorale à la fin du 19ème siècle », Actes de la recherche en sciences sociales, $\mathrm{n}^{\circ}$ 47-48, p. 90-112. 
Le Cozanet Laurène (2019), La professionnalisation des études. Quand l'Université (s')adapte à l'économie, thèse de science politique, université Paris Dauphine.

Losego Philippe (2004), «Le travail invisible à l'université : le cas des antennes universitaires », Sociologie du travail, vol. 46, p. 187-204.

Louvel Séverine (2011), Des patrons aux managers. Les laboratoires de la recherche publique depuis les années 1970. Rennes, Presses universitaires de Rennes.

Louvel Séverine \& Valette Annick (2014), «Les carrières à l'université. Une approche par les modes d'engagement dans la promotion », Revue d'anthropologie des connaissances, ${ }^{\circ} 3$, p. 523-546.

Ministère de l'Enseignement supérieur, de la recherche et de l'innovation (2019). État de l'Enseignement supérieur, de la Recherche et de l'Innovation.

Musselin Christine (2015), « Peut-on parler d'égalité des chances dans les carrières universitaires en France ?», Regards croisés sur l'économie, n 16, p. 203-217.

Musselin Christine (2017), La grande course des universités, Paris, Presses de Sciences Po.

Paradeise Catherine (2011), «La profession académique saisie par la nouvelle gestion publique : le cas français », Sociologie du travail, vol. 53, $\mathrm{n}^{\circ} 3$.

Paye Simon (2015). «Temporaires, permanents, « vedettes » : la tripartition du salariat académique au Royaume-Uni ». Sociologie du Travail, vol. 57, n², p.175-199.

Picard Emmanuelle (2012). «Les enseignants-chercheurs : une évaluation centralisée. Du comité consultatif de l'enseignement supérieur au CNU (1873-1992)». Spirales, n49, p.69-82.

Picard Emmanuelle (2020), La profession introuvable ? Les universitaires français de l'Université impériale aux universités contemporaines, mémoire inédit d'HDR, université Paris 1 Panthéon Sorbonne ; en ligne : https://halshs.archives-ouvertes.fr/tel-02498327.

Picard Jean-François (1990), La République des Savants. La recherche française et le CNRS, Flammarion.

Pontille David \& Torny Didier (2012), « Rendre publique l'évaluation des SHS : les controverses sur les listes de revues de l'AERES ». Quaderni, n 77, p. 11-24.

Prost A. \& Cytermann J.-R. (2010), « Une histoire en chiffres de l'enseignement supérieur en France », Le Mouvement Social, $\mathrm{n}^{\circ}$ 233, p. 31-46.

Renisio Yann (2015). «L'origine sociale des disciplines ». Actes de la recherche en sciences sociales, $\mathrm{n}^{\circ} 210$, p. $10-27$.

Rogers Rebecca \& Molinier Pascale (dir.) (2016). Les femmes dans le monde académique. Rennes, PUR.

Schwartz Rémy (2008). Commission de réflexion sur l'avenir des personnels de l'Enseignement supérieur. Rapport pour le Ministère de l'Enseignement supérieur et de la Recherche

Verschueren Pierre (2017). Des savants aux chercheurs. Les sciences physiques comme métier (France, 1945-1968). Thèse d'histoire, université Paris 1 Panthéon-Sorbonne. 